\title{
PRODUÇÃO DE SERAPILHEIRA E APORTE DE NUTRIENTES DE ESPÉCIES ARBÓREAS NATIVAS EM UM SISTEMA AGROFLORESTAL NA ZONA DA MATA DE MINAS GERAIS ${ }^{1}$
}

\author{
Catalina Jaramillo-Botero ${ }^{2}$, Ricardo Henrique Silva Santos ${ }^{3}$, Merci Pereira Fardim ${ }^{4}$, Thelma Mendes \\ Pontes ${ }^{5}$ e Flávia Sarmiento 4
}

\begin{abstract}
RESUMO - O objetivo deste trabalho foi avaliar a deposição de serapilheira de espécies arbóreas nativas da Mata Atlântica e o aporte potencial de nutrientes em um sistema agroflorestal (SAF). No local do experimento foram determinados 40 pontos amostrais nas entrelinhas do cafezal, nos quais foram colocados coletores de serapilheira. As folhas depositadas foram coletadas a cada mês, durante um ano, pesadas e separadas por espécie. Foram coletadas folhas frescas e senescentes das espécies arbóreas do SAFe determinado o seu teor de macronutrientes. Entre as espécies estudadas, a cutieria (Joannesia princeps Vell.) apresentou a mais alta produção de serapilheira (76,41 kg MS indivíduo ${ }^{-1}$ ano $^{-1}$ ), seguida de capixingui (Croton floribundus Spreng.), guapuruvu (Schizolobium parahyba Vell.) e fedegoso (Senna macranthera (Collad)). As árvores de cutieira e capixingui apresentaram maior queda de folhas em fevereiro, fedegoso em novembro e guapuruvu em julho. A cutieira destacou-se pela alta capacidade de aporte de nitrogênio (438,5 $\mathrm{g}_{\text {indivíduo }}^{-1}$ ano $\left.^{-1}\right)$ e o capixingui de cálcio (581 g indivíduo${ }^{1}$ ano $\left.^{-1}\right)$ e potássio $\left(299,7 \mathrm{~g}_{\text {indivíduo }}{ }^{-1} \mathrm{ano}^{-1}\right)$. Apesar do alto teor de nitrogênio nas folhas frescas de fedegoso $(2,83 \%)$, a alta translocação e a baixa deposição de serapilheira fizeram com que o aporte potencial de nitrogênio fosse baixo, em comparação com as outras espécies. As árvores de capixingui e cutieira apresentam grande potencial de aporte de nutrientes ao sistema e, mais especificamente aos cafeeiros, durante a época em que a cultura apresenta alta demanda nutricional.
\end{abstract}

Palavras-chave: Joannesia princeps, Croton floribundus e Schizolobium parahyba.

\section{LITTER PRODUCTION AND POTENTIAL NUTRIENT INPUT OF NATIVE TREE SPECIES IN AN AGROFORESTRY SYSTEM AT ZONA DA MATA, MG, BRAZIL}

\begin{abstract}
This work aimed to study litter fall deposition of native species from the Atlantic Forest and its potential nutrient input in the agroforestry system. Forty sampling points were determined in the experimental site between the coffee rows where litter collectors were placed. The deposited leaves were collected each month over a year, selected by species, and their dry weight was determined. Fresh and senescent leaves were also collected and their macronutrient was also determined. Among the studied species, cutieira (Joannesia

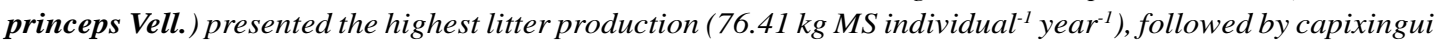
(Croton floribundus Spreng.), guapuruvu (Schizolobium parahyba Vell.) and fedegoso (Senna macranthera (Collad).). Cutieira and capixingui trees showed the highest leaffall in February, fedegoso in November and guapuruvu in July. Cutieira showed high nitrogen input capacity (438.5 $\mathrm{g}_{\text {tree }}^{-1}$ year-1), capixingui showed high calcium (581 $\mathrm{g} \mathrm{tree}^{-1}$ year $\left.{ }^{-1}\right)$ and potassium (299.7 $\mathrm{g} \mathrm{tree}^{-1}$ year $\left.^{-1}\right)$. Despite the high nitrogen content in the fedegoso fresh leaves (2.83\%), high translocation and low litter fall resulted in small nitrogen input compared to the other species. Capixingui and cutieira showed high potential of nutrient input to the agroforestry system, and in particular, to coffee plants, during the highest nutrient demand period.
\end{abstract}

Keywords: Joannesia princeps, Croton floribundus and Schizolobium parahyba.

\footnotetext{
${ }^{1}$ Recebido em 12.02.2007 e aceito para publicação em 22.08.2008.

${ }^{2}$ Programa de Pós-Graduação em Fitotecnia da Universidade Federal de Viçosa (UFV). E-mail:<catilinjara@yahoo.com>

${ }^{3}$ Departamento de Fitotecnia da UFV. E-mail: <rsantos@ufv.br>.

${ }^{4}$ Graduando em Agronomia da UFV>. E-mail: <mercipfardin@ @ahoo.com.br>.

${ }^{5}$ Programa de Pós-Graduação em Ecologia do Instituto Nacional de Pesquisa do Amazonas (INPA).E-mail:<thelma_pontes@hotmail.com>
} 


\section{INTRODUÇÃO}

Emregiões que possuem solos de baixa fertilidade, elevada acideze topografia montanhosaé recomendada a implantação de sistema agroflorestal (SAF), uma vez que estes sistemas podem recuperar e até incrementar a fertilidade dos solos (MENDONÇAe STOTT, 2003). Entre outras vantagens dos SAFs se encontram a capacidade de regular o ciclo hídrico (GIRALDO e JARAMILLO, 2004), aumentar a captura de dióxido de carbono e aumentar a biodiversidade local (PERFECTO et al., 2005).

No caso dos SAFs com café, além dos benefícios ecológicos, o café proveniente destes sistemas pode ser comercializado dentro do grupo dos cafés especiais, com preços melhores e mais estáveis que os apresentados no mercado convencional (PERFECTO et al., 2005). A segurança no mercado beneficia economicamente as comunidades envolvidas na produção de café sob SAF e ajuda a melhorar as condições socioeconômicas da região (BACON, 2005).

Um dos benefícios ecológicos da introdução de árvores nas lavouras de café é a produção de serapilheira (HAIRIAH et al., 2006). A cobertura morta evita a incidência da radiação solar direta sobre o solo, conserva a umidade e contribui com a ciclagem de nutrientes. Quando usadas árvores leguminosas, além do aporte de matéria orgânica, estas podem contribuir com o incremento do nitrogênio no solo (SCHROTH et al., 2001).

Em países como Costa Rica, México e Colômbia nos quais os SAFs com café são altamente difundidos, são freqüentemente utilizadas espécies arbóreas leguminosas como Gliricidia sepium (Jacq.), Inga edulis Mart. e Erytrhina poeppigiana Walpers. Nas pesquisas desenvolvidas, estas espécies têm apresentado alto conteúdo de nitrogênio foliar, rápida decomposição (SCHWENDENER et al., 2005), alta capacidade de fixação de nitrogênio atmosférico (LEBLANC et al., 2005) e grande aporte de nitrogênio a partir dos nódulos e da decomposição de biomassa aérea depositada na serapilheira (NYGREN e RAMÍREZ, 1995). Além do nitrogênio, outros nutrientes podem ser melhor aproveitados uma vez que as árvores podem explorar camadas mais profundas do solo e contribuir com a ciclagem de nutrientes (GINDABA et al., 2005). No entanto, algumas espécies arbóreas possuem mecanismos que permitem aproveitar os recursos limitados para o seu próprio beneficio, em detrimento da cultura. Neste caso a capacidade de competição com a cultura vai depender da sincronia dos estádios fenológicos das espécies associadas e das práticas de manejo como o distanciamento e a poda (LIVESLEY et al., 2000).

No Brasil, na Zona da Mata de Minas Gerais existem experiências com SAFs em que os cafeeiros são frequentemente plantados junto com árvores frutíferas, principalmente abacate (Persea americana Mill.) e frutas cítricas (Citrus spp.). Também são usadas espécies leguminosas de rápido crescimento, como guapuruvu (Schizolobium parahyba (Vell.) Toledo, angico (Anadenanthera columbrina (Vell.) Brenan e fedegoso (Senna macranthera (Colladon) Irwin \& Barneby). Em alguns casos os agricultores plantam árvores para produção de madeira, entre elas cedro toona (Toona ciliata M. Roem), candeia (Vanillosmopsis erythropapa Schult.) e ipê amarelo (Tabebuia ochracea (Cham.) Standl.). Também se observam espécies arbóreas típicas de estádios iniciais de sucessão nas florestas semideciduais, como embaúba (Cecropia hololeuca Miq.), jacaré (Piptadenia gonoacantha Mart.) cutieira (Joannesia princeps Vell.) e capixingui (Croton floribundus Spreng.) (SOUZA et al., 2006).

Existe grande controvérsia sobre a escolha das espécies arbóreas mais apropriadas para serem usadas nos SAFs com café nesta região. As observações dos agricultores geram opiniões contraditórias e os pesquisadores contam com pouca informação científica sobre o comportamento das espécies em sistemas agroflorestais. É necessário conhecer os potenciais benefícios e prejuízos das espécies arbóreas associadas com os cafeeiros, que tradicionalmente são cultivados a pleno sol na região.

O objetivo deste trabalho foi avaliar a deposição de serapilheira de espécies arbóreas nativas da Mata Atlântica e o aporte potencial de nutrientes em um SAF na Zona da Mata de Minas Gerais.

\section{MATERIAL E MÉTODOS}

\subsection{Características do local}

O experimento foi conduzido entre agosto de 2005 e julho de 2006, em um sistema agroflorestal com café com 14 anos de idade, na propriedade do agricultor Ilson José de Medeiros Lopes. A área experimental está localizada no município de Araponga (MG, Brasil), dentro da área de amortecimento do Parque Estadual da Serra do Brigadeiro, a 20 40' 11,9' Sul e 42 33' 11,8' Oeste, com altitude de $990 \mathrm{~m}$. O terreno tem 28,5\% de declividade e está voltado para o norte. O espaçamento entre cafeeiros (Coffea arabica cv. catuaí vermelho) 
é 3 x 1,50 m e as espécies arbóreas se encontram plantadas nas entrelinhas.

A temperatura média anual é de $20^{\circ} \mathrm{C}$, com média de $16^{\circ} \mathrm{C}$ no inverno (julho até agosto) e $23^{\circ} \mathrm{C}$ no verão (dezembro até março). A precipitação média anual é de $1109 \mathrm{~mm}$ com um período seco entre os meses de abril a setembro (Figura 1).

O solo, classificado como Latosolo Vermelho Amarelo, apresenta as seguintes características: $\mathrm{pH}$ $\left(\mathrm{H}_{2} \mathrm{O}\right) 5,6 ; \mathrm{P}_{2} \mathrm{O}_{5} 3,7 \mathrm{mg} \mathrm{kg}^{-1} ; \mathrm{K}_{2} \mathrm{O} 62 \mathrm{mg} \mathrm{kg}^{-1}$; $\mathrm{Ca} 3,2 \mathrm{cmol}$ $\mathrm{kg}^{-1}$; Mg 0,8 cmol kg-1 , CTC (t) 4,16 cmol kg-1, na profundidade de $0-20 \mathrm{~cm}$.

Segundo informação do agricultor, ao longo dos 11 anos a plantação tem sido adubada com esterco ou adubo mineral. No ano anterior ao início da pesquisa e durante o período avaliado, os cafeeiros foram adubados com $100 \mathrm{~g} \mathrm{cova}^{-1} \mathrm{da}$ formulação 20-0-20 (N-P-K).

\subsection{Componente arbóreo e a cultura}

As espécies arbóreas encontradas no SAF foram: guapuruvu (Schizolobium parahyba), fedegoso (Senna macranthera), cutieira (Joannesia princeps), angico (Anadenanthera colubrina) e capixingui (Croton floribundus). O critério de inclusão dos indivíduos foi o diâmetro a altura do peito (DAP) igual ou maior a $10 \mathrm{~cm}$. Cada indivíduo foi marcado e identificado e medidos o diâmetro a altura do peito $(1,30 \mathrm{~m})$ e a altura. Foi determinado o número de indivíduos de cada espécie e a densidade relativa, segundo Martins (1991) (Quadro 1). Foram selecionadas 40 plantas de café distribuídas de 0 a $7 \mathrm{~m}$ das árvores do sistema.

A altura e a distribuição das árvores e das plantas de café marcadas foram determinadas por meio do levantamento topográfico planialtimétrico, utilizando a estação total Topicum gts $212 ®$. Os dados foram georeferenciados e sistematizados com ajuda do programa ArcView ${ }^{\circledR}$.

\subsection{Amostragem e análises da serapilheira}

A queda de serapilheira das árvores foi determinada por coletas mensais segundo a metodologia modificada por Campanha et al., (2004). Telas de nylon de $0,25 \mathrm{~m}^{2}$ foram colocadas sobre o solo nas entrelinhas próximo a cada cafeeiro marcado. Mensalmente foi retirada a massa de folhas e separada por espécie. A serapilheira proveniente do angico não foi avaliada devido à dificuldade para coletar os pequenos folíolos característicos desta espécie. O material coletado foi submetido à secagem em estufa de ventilação de ar forçada a $70^{\circ} \mathrm{C}$ até massa constante. A produção média mensal de serapilheira de cada espécie foi dividida pelo número de indivíduos presentes no $\mathrm{SAF}$, para determinar a produção por indivíduo por mês (kg MS indivíduo ${ }^{-1}$ mês $\left.^{-1}\right)$. Foram somados os valores mensais de produção por indivíduo ao longo do ano, para determinar a produção de serapilheira por indivíduo por ano (kg MS indivíduo ${ }^{-1} \mathrm{ano}^{-1}$ ).

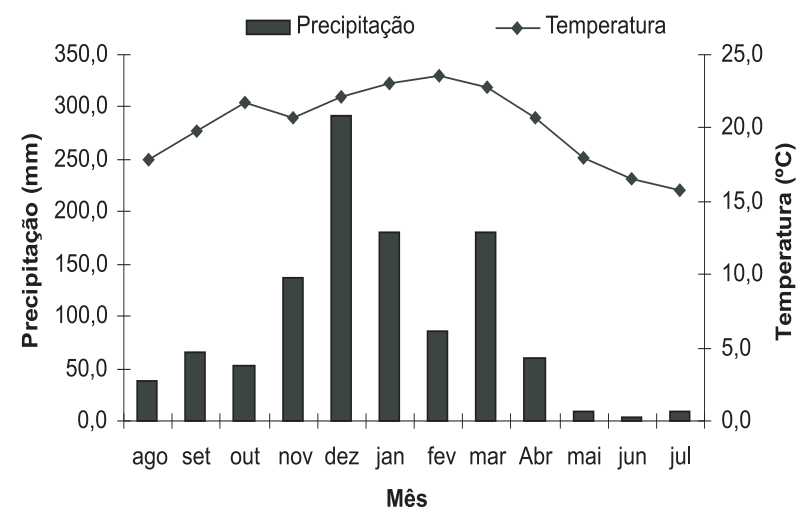

Figura 1 - Variação da precipitação e da temperatura na região de estudo durante o desenvolvimento do experimento (agosto 2005 - julho 2006).

Figure 1 - Rainfall and temperature distribution at the region under study during the experimental period (August 2005- July 2006).

Quadro 1 - Número total de indivíduos por espécie, densidade relativa, diâmetro a $1,30 \mathrm{~m}$ (DAP) e altura de guapuruvu ( $S$. parahyba), fedegoso ( $S$. macranthera), angico (Anadenanthera columbrina), capixingui (C. floribundus) e cutieira (J. princeps) em sistema agroflorestal na Zona da Mata de Minas Gerais

Table 1 - Total number of indivuduals per species, relative density, diameter at 1.30m (DAP) and height of guapuruvu (S. parahyba), fedegoso (S. macranthera), angico (Anadenanthera columbrina), capixingui (C. floribundus) e cutieira (J. princeps) in an agroforestry system at Zona da Mata, Minas Gerais, Brazil

\begin{tabular}{lcccc}
\hline Espécie & $\begin{array}{c}\text { Número } \\
\text { indivíduos }\end{array}$ & $\begin{array}{c}\text { Densidade } \\
\text { Relativa }\end{array}$ & $\begin{array}{c}\text { DAP } \\
\mathrm{cm}\end{array}$ & $\begin{array}{c}\text { Altura } \\
\mathrm{m}\end{array}$ \\
\hline Guapuruvu & 17 & 47,2 & 74 & 10 \\
Fedegoso & 12 & 33,3 & 85 & 7 \\
Angico & 3 & 8,3 & 41 & 7 \\
Cutieira & 3 & 8,3 & 92 & 8 \\
Capixingui & 1 & 2,8 & 56 & 8 \\
\hline
\end{tabular}

R. Árvore, Viçosa-MG, v.32, n.5, p.869-877, 2008 
Para calcular o aporte potencial de nutrientes de cada espécie arbórea a cada mês foram coletadas folhas recém caídas de cada espécie e misturadas para formar uma única amostra composta. Este procedimento permitiu eliminar o efeito da translocação de nutrientes antes da queda das folhas, evitando superestimar o aporte de nutrientes das árvores via serapilheira.

Para determinar os nutrientes nas folhas, foi realizada digestão nitricoperclórica (JOHNSON e ULRICH, 1959), determinado o teor de P (BRAGA e DEFELIPO, 1974), $\mathrm{K}, \mathrm{Ca}, \mathrm{Mg}$ (AOAC, 1975) e N total (método de destilação de Kjehldahl). O conteúdo de nutrientes foi calculado multiplicando o teor de cada nutriente nas folhas de cada espécie pela quantidade de massa seca depositada, sendo expresso em g indivíduo ${ }^{-1} \mathrm{mês}^{-1}$.

\section{RESULTADOS E DISCUSSÃO}

\subsection{Produção de massa}

Entre as árvores avaliadas no sistema agroflorestal a cutieira foi a que apresentou o mais alto valor de produção de serapilheira com 76,41 kg MS indivíduo${ }^{1}$ ano $^{-1}$, seguida em ordem decrescente por capixingui $\left(57,88 \mathrm{~kg}\right.$ MS indivíduo ${ }^{-1}$ ano $\left.^{-1}\right)$, guapuruvu $(19,09 \mathrm{~kg}$ MS indivíduo ${ }^{-1}$ ano $\left.^{-1}\right)$ e fedegoso $\left(16,17 \mathrm{~kg} \mathrm{MS}^{-1}\right.$ indivíduo ${ }^{-1}$ ano $\left.^{-1}\right)$. Os valores de queda de serapilheira apresentados por estas espécies são muito superiores aos registrados por araribá (Centrolobium tomentosum), em floresta semidecídua no Estado de São Paulo, que apresentou 4,2 kg MS indivíduo ${ }^{-1}$ ano $^{-1}$ incluindo folíolos, raques, flores e frutos (AIDAR e JOLY, 2003). A produção de serapilheira total anual proveniente de folhas observada no SAF foi de 5,2 $\mathrm{Mg} \mathrm{ha}^{-1} \mathrm{ano}^{-1}$. Em outro trabalho na região de Viçosa, foi encontrada a produção de $6,8 \mathrm{Mg} \mathrm{ha}^{-1} \mathrm{ano}^{-1}$ de fração foliar (ARATO et al., 2003). Possivelmente a diferença de aporte de material esteja relacionada com o fato das espécies estudadas serem tipicamente pioneiras e de rápido crescimento. As espécies típicas de estádios de sucessão primária nas florestas se caracterizam por fazer grande investimento na produção de biomassa em curto espaço de tempo (MARTINS e RODRIGUES, 1999).

A queda sazonal de folhas das árvores presentes no SAF variou de acordo com a espécie. As árvores de guapuruvu apresentaram o principal pico de queda de folhas em julho (33,2 \% MS produzida no ano por esta espécie) e agosto (22,9\% MS) (Figura 2), coincidindo com os meses em que se apresentaram baixas temperaturas e baixa precipitação (Figura 1). Este comportamento é típico nas florestas estacionais semideciduais e tem sido observado em florestas de Minas Gerais na região de Belo Horizonte (PAULA e LEMOS FILHO, 2001) e Viçosa (ARATO et al., 2003). A perda de folhas das árvores durante a estação seca é conhecida como um mecanismo de resposta ao estresse hídrico, para reduzir a perda de água por transpiração (MARTINS e RODRIGUES, 1999).

As árvores de fedegoso e cutieira apresentaram padrões similares de queda de folhas ao longo do ano todo. Houve picos de queda mais intensa durante a estação chuvosa e quente, nos meses de novembro e fevereiro (Figura 2). No mês de fevereiro o capixingui apresentou o pico de maior produção de serapilheira.

As plantas de café apresentaram uma dinâmica sazonal similar à das árvores de guapuruvu, com grande queda de folhas durante o inverno. A maior queda de folhas se apresentou no mês de junho, com 30,3\% do total de folhas depositadas ao longo do ano, equivalente a 147 , $5 \mathrm{~g}$ de MS de folhas por planta de café (Figura 2). A perda das folhas dos cafeeiros evita a perda excessiva de água causada pela transpiração. Durante esta época o crescimento das plantas de café é mínimo (SILVA et al., 2004). A serapilheira do guapuruvu e dos cafeeiros que é depositada durante o inverno pode reduzir a evaporação da água nas camadas superiores do solo, ajudando a diminuir a magnitude do estresse hídrico suportado pelos cafeeiros. Em sistema agroflorestal, no trópico semi-árido, a serapilheira reduziu a perda de água do solo por evaporação (KINAMA et al., 2005).

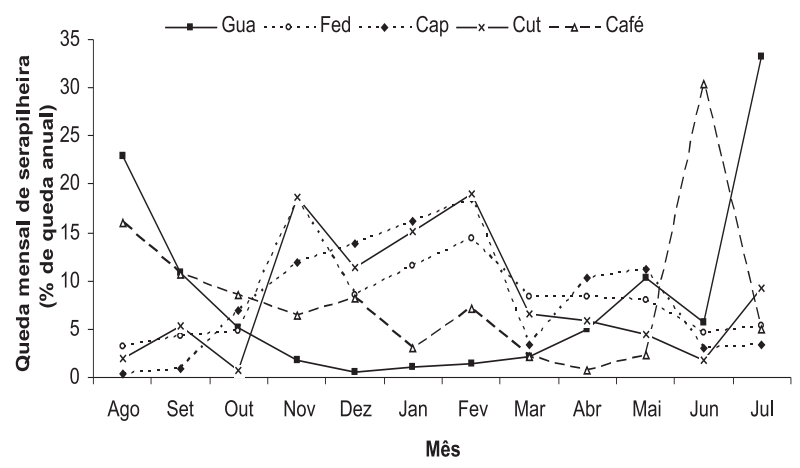

Figura 2-Distribuição da queda de serapilheira de guapuruvu ( $S$. parahyba), fedegoso (S. macranthera), capixingui ( $C$. floribundus), cutieira (J.princeps) e café (C. arabica) ao longo do período de estudo (2005 - 2006).

Figure 2 - Litter fall distribution of guapuruvu (S. parahyba), fedegoso(S. macranthera), capixingui (C. floribundus), cutieira (J. princeps) and coffee (C. arabica) during the study period (August 2005-July 2006). 


\subsection{Teor de nutrientes nas folhas}

As folhas frescas de fedegoso apresentaram o mais alto teor de nitrogênio, seguidas das folhas de capixingui (Quadro 2). Em folhas frescas de árvores leguminosas é comum encontrar alto teor de nitrogênio. Como exemplo tem Leucaena leucocephala (Lam.) que é uma espécie bem conhecida pela sua alta eficiência para fixar nitrogênio atmosférico e pelo alto conteúdo de nitrogênio nas folhas, que chegam a apresentar 3,3\% MS de nitrogênio (PARROTA, 1999). Outras espécies leguminosas como Albizzia lebbeck (Benth.) e Dalbergia sisso Roxb. apresentam em média 2,63\% e 2,19\% de MS de N (SEMWAL et al., 2003). Leguminosas nativas de regiões tropicais como a Casuarina equisetifolia L. ou Stryphnodendron microstachyum Poepp. \& Endl., apresentam menor teor de nitrogênio foliar $1,8 \% \mathrm{MS}$ (PARROTA, 1999) e 1,94\% MS respectivamente (BYARD et al., 1996). Estes exemplos indicam que as espécies estudadas possuem níveis intermédios de teor de nitrogênio nas folhas, inclusive quando se trata de espécies não leguminosas como a cutieira.

Quadro 2 - Teor de nutrientes presentes em folhas frescas e senescentes de guapuruvu (S. parahyba), fedegoso ( $S$. macranthera), capixingui (C. floribundus) e cutieira (J.princeps) e retranslocação de nutrientes (\% do teor inicial de nutrientes)

Table 2 - Nutrient content of fresh and senescent leaves of guapuruvu (S. parahyba), fedegoso (S. macranthera), capixingui (C. floribundus) and cutieira (J. princeps) and nutrient retranslocation (\% of the initial nutrient content)

\begin{tabular}{ccccc}
\hline Material & \multicolumn{4}{c}{ Teor de nutrientes (\%MS) } \\
\cline { 2 - 5 } vegetal & Guapuruvu & Fedegoso & Capixingui & Cutieira \\
\hline Folhas Frescas & & & & \\
$\mathrm{N}$ & 2,27 & 2,83 & 2,60 & 2,21 \\
$\mathrm{P}$ & 0,18 & 0,28 & 0,27 & 0,29 \\
$\mathrm{~K}$ & 0,67 & 0,99 & 1,57 & 1,12 \\
$\mathrm{Ca}$ & 1,49 & 1,51 & 1,63 & 1,16 \\
$\mathrm{Mg}$ & 0,32 & 0,26 & 0,38 & 0,44 \\
Folhas Senescentes & & & & \\
$\mathrm{N}$ & 1,88 & 1,96 & 0,62 & 1,20 \\
$\mathrm{P}$ & 0,17 & 0,14 & 0,14 & 0,05 \\
$\mathrm{~K}$ & 0,67 & 0,72 & 1,15 & 0,67 \\
$\mathrm{Ca}$ & 1,02 & 1,51 & 1,35 & 0,98 \\
$\mathrm{Mg}$ & 0,21 & 0,26 & 0,36 & 0,38 \\
Retranslocação & & & & \\
$\mathrm{N}$ & 17,18 & 30,74 & 76,15 & 45,70 \\
$\mathrm{P}$ & 5,56 & 50,00 & 48,15 & 82,76 \\
$\mathrm{~K}$ & 0,00 & 27,27 & 26,75 & 40,18 \\
$\mathrm{Ca}$ & 31,54 & 0,00 & 17,18 & 15,52 \\
$\mathrm{Mg}$ & 34,38 & 0,00 & 5,26 & 13,64 \\
\hline
\end{tabular}

O teor de nitrogênio na serapilheira foi menor que o observado nas folhas frescas devido a retranslocação de nutrientes (Quadro 2), que ocorre das folhas senescentes para outros órgãos da plantas, antes das folhas caírem (LODHIYAL et al., 2002). Fedegoso e cutieira apresentaram valores de retranslocação de nitrogênio próximos a os encontrados por Lodhiyal e Lodhiyal (2003) em árvores de Dalbergia sissoo, que variaram entre 34,8 e 37,3\%. O guapuruvu apresentou baixa porcentagem de retranslocação de nitrogênio $(17,18 \%)$, o que permitiu a manutenção de alto teor de nitrogênio nas folhas senescentes. $O$ alto valor de retranslocação apresentado pelas árvores de fedegoso e cutieira pode ser devido em parte ao alto teor de nutrientes presentes nas folhas, que segundo Lodhiyal e Lodhiyal (1997) está relacionado com a maior capacidade de translocação de nutrientes.

A alta translocação de fósforo observada na cutieira se destaca dos valores apresentados pelas outras espécies. Isto explica em parte o baixo teor de fósforo nas folhas senescentes desta espécie. Os teores de fósforo e potássio nas folhas senescentes de guapuruvu, fedegoso e capixingui foram similares ao da serapilheira proveniente de Albizzia lebbek em bosque montano no Himalaya (SEMWAL, 2003) e mais alto que o apresentado por Centrolobium tomentosum Guill. ex. Benth, em mata ciliar em São Paulo (AIDAR e JOLI, 2003) e de Araucaria angustifolia (Bertol) em Rio grande do Sul (SCHUMACHER et al., 2004).

O capixingui apresentou alto teor de potássio, cálcio e magnésio, comparados com os valores apresentados pelas outras espécies. Estes valores foram similares aos observados em mudas desta espécie tratadas com águas residuais provenientes de esgoto doméstico (AUGUSTO et al., 2003).

\subsection{Aporte de nutrientes}

A grande produção de serapilheira da cutieira e o alto teor médio de nitrogênio nas folhas, fez com que seu potencial de aporte de nitrogênio ao sistema fosse superior que o das outras espécies. Além destas características, a sincronia entre a alta deposição de serapilheira e a época de maior demanda de nutrientes dos cafeeiros, faz dela uma espécie interessante para ser usada nos SAF com café da região. Contudo outras características das folhas como o teor de lignina, polifenóis e taninos devem ser estudados em profundidade, uma vez que afetam a velocidade de liberação dos nutrientes

R. Árvore, Viçosa-MG, v.32, n.5, p.869-877, 2008 
e a capacidade de serem disponibilizados para a cultura (MUSVOTO et al., 2000). No caso das folhas de cutieira apresentarem teores elevados destes compostos, o possível aporte de $\mathrm{N}$ ao sistema seria reduzido.

Durante os meses de julho e agosto o guapuruvu também se destacou pelo aporte de nitrogênio com valores de 88,8 e 61,3 g de $\mathrm{N}$ indivíduo. O maior aporte de nitrogênio do fedegoso foi observado no mês de novembro com $43 \mathrm{~g}$ indivíduo ${ }^{-1}$ de N (Figura 3). Não foram encontradas referências sobre a capacidade de nodulação ou de fixação biológica de nitrogênio nestas espécies leguminosas.

Outras espécies leguminosas características de florestas estacionais semideciduais como Centrolobium tomentosum e Anadenanthera falcata (Benth.) apresentam valores inferiores aos observados no experimento, com aporte anual de nitrogênio de 54,3 $\mathrm{g}$ indivíduo ${ }^{-1}$ para C. tomentosum e $34,0 \mathrm{~g}$ indivíduo${ }^{1}$ para A. falcata (AIDAR e JOLI, 2003).

O aporte de fósforo proveniente da serapilheira foi baixo comparado com os valores observados para os outros nutrientes (Figura 4). Como observado no Quadro 2, o teor de fósforo nas folhas de todas as espécies foi baixo comparado com os outros nutrientes, isto explica o baixo aporte deste elemento por parte da serapilheira ao sistema.

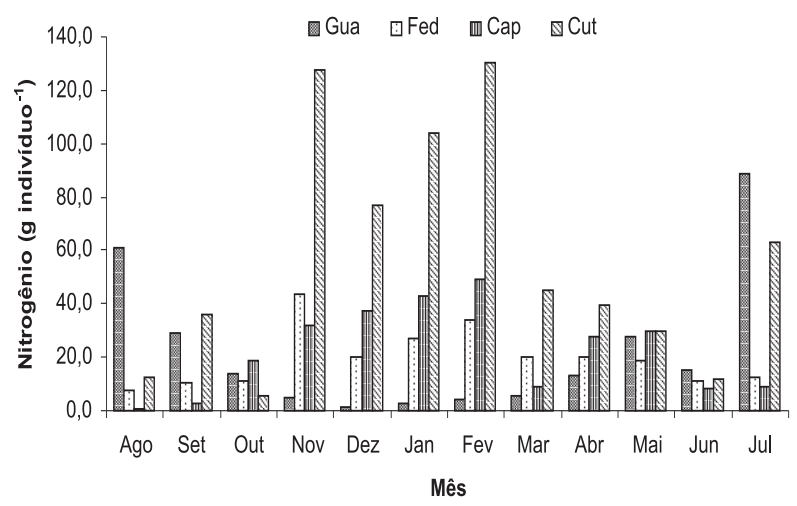

Figura 3 - Aporte de nitrogênio proveniente das folhas de guapuruvu ( $S$. parahyba), fedegoso ( $S$. macranthera), capixingui (C.floribundus) e cutieira (J. princeps) depositadas no solo ao longo de um ano (agosto 2005- julho 2006).

Figure 3 - Nitrogen input from leaves of guapuruvu ( $S$. parahyba), fedegoso (S. macranthera), capixingui (C. floribundus) and cutieira (J. princeps) deposited on soil over one year (August 2005-July 2006).

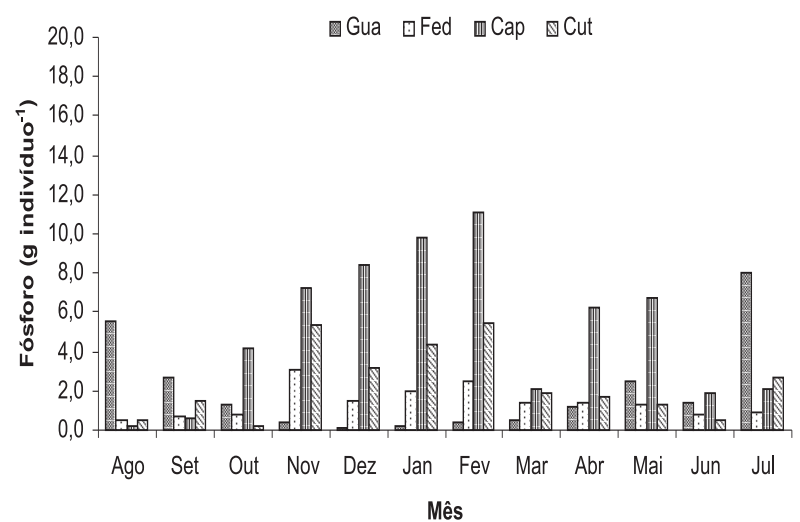

Figura 4 -Aporte de fósforo proveniente das folhas de guapuruvu (S. parahyba), fedegoso (S. macranthera), capixingui (C. floribundus) e cutieira (J. princeps) depositadas no solo ao longo de um ano ( $\mathrm{g}_{\text {indivíduo }}{ }^{-1}$ ).

Figure 4-Phosphorus input from leaves of guapuruvu ( $S$. parahyba), fedegoso (S. macranthera), capixingui (C.floribundus) and cutieira (J. princeps) deposited on soil over one year (August 2005-July 2006).

A baixa produção de serapilheira do fedegoso fez com que o aporte de fósforo ao sistema fosse baixo, apesar do teor relativamente alto de fósforo apresentado nas folhas (Quadro 2). O fedegoso é uma espécie que tem demonstrado ser altamente adaptada a condições de solos pobres em fósforo (FLORES-AYLAS et al., 2003). Esta é uma característica interessante para ser explorada nos SAF, uma vez que existe a possibilidade de que o fedegoso apresente baixa competição por fósforo e ainda, contribua com a ciclagem deste nutriente.

Capixingui e cutieira apresentaram grande aporte de potássio por indivíduo nos meses de novembro, dezembro, janeiro e fevereiro (Figura 5). O valor acumulado para estes meses foi de $299,7 \mathrm{~g}$ indivíduo ${ }^{-1}$ para capixingui e $244,7 \mathrm{~g}^{\text {indivíduo }}{ }^{-1}$ para cutieira. $\mathrm{O}$ alto aporte de potássio coincide com os meses de maior demanda deste nutriente por parte dos cafeeiros, tornando estas espécies boas opções para consorciar com esta cultura.

As árvores de capixingui e cutieira apresentaram grande aporte potencial de cálcio (equivalente a 581 e $557 \mathrm{~g}$ indivíduo $^{-1} \mathrm{ano}^{-1}$ respectivamente) e magnésio (155 e $216 \mathrm{~g}_{\text {de }} \mathrm{Mg}$ indivíduo ${ }^{-1} \mathrm{ano}^{-1}$ respectivamente) $^{-1}$ através da serapilheira (Figuras 6 e 7). Estes valores são muito superiores (3,5 e 3 vezes respectivamente) aos apresentados por Metrodorea nigra A. St.-Hil., em floresta estacional semidecidual de terras baixas no estado do Rio de Janeiro (VILELA et al., 2006). 


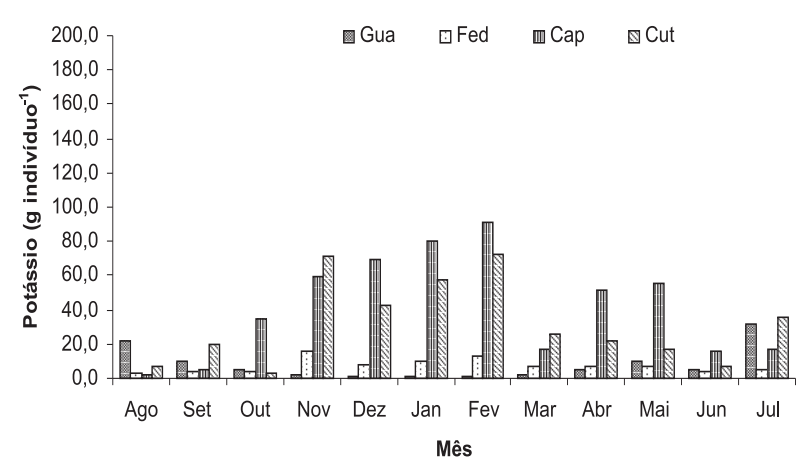

Figura 5-Aporte de potássio proveniente das folhas de guapuruvu (S. parahyba), fedegoso (S. macranthera), capixingui (C. floribundus) e cutieira (J. princeps) depositadas no solo ao longo de um ano (agosto 2005 - julho 2006).

Figure 5 - Potassium input from leaves of guapuruvu ( $S$. parahyba), fedegoso (S. macranthera), capixingui (C. floribundus) and cutieira (J. princeps) deposited on soil over one year (August 2005-July 2006).

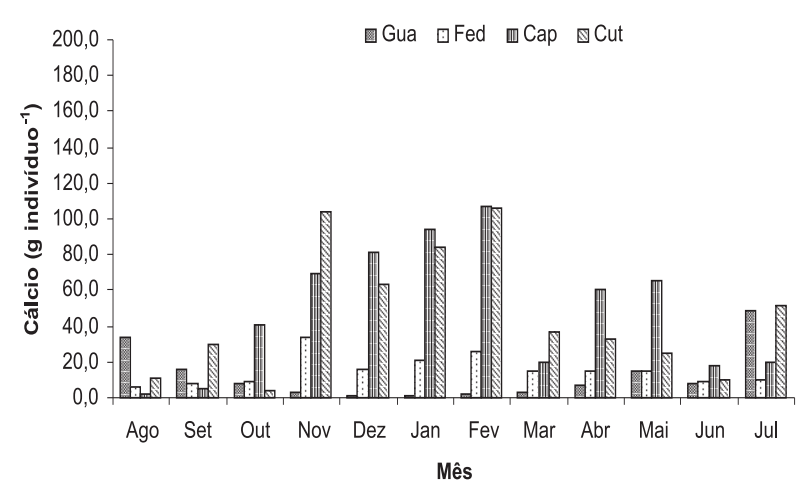

Figura 6-Aporte de cálcio proveniente das folhas de guapuruvu (S. parahyba), fedegoso (S. macranthera), capixingui (C. floribundus) e cutieira (J. princeps) depositadas no solo ao longo de um ano (agosto 2005 - julho 2006).

Figure 6-Calcium input from leaves of guapuruvu (S. parahyba), fedegoso (S. macranthera), capixingui (C.floribundus) and cutieira (J. princeps) deposited on soil over one year (August 2005-July 2006).

Os resultados apresentados mostram a alta capacidade das árvores de capixingui de ciclarem nutrientes através da serapilheira. Outras características da espécie reportadas por Gonçalves (2000) são a alta taxa de crescimento, alta demanda de nutrientes e alta capacidade de absorção e acúmulo destes nos tecidos vegetais. Estas características podem fazer com que esta espécie apresente alta competição com os cafeeiros pelos nutrientes disponíveis no solo. No entanto outra espécie do mesmo gênero, Croton macrostachyus Hochst.\&Rich, tem apresentado grande capacidade de translocar nutrientes das camadas profundas do solo para a superfície, principalmente potássio e fósforo (GINDABA et al., 2005).

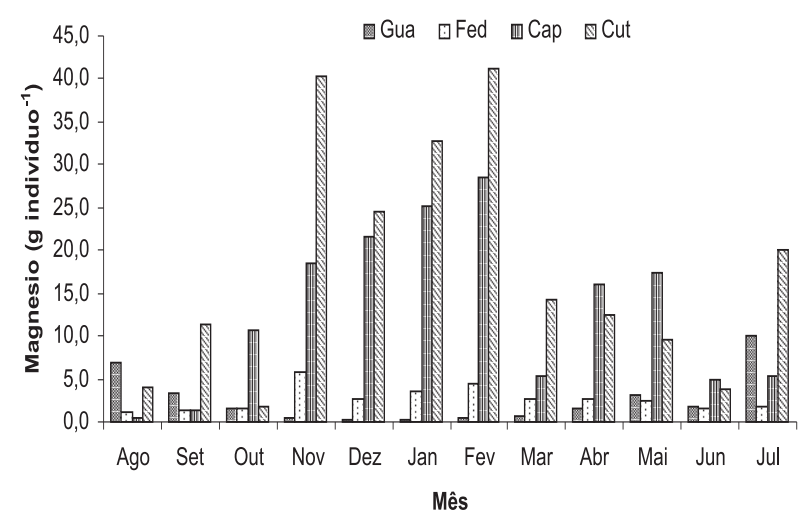

Figura 7 - Aporte de magnésio proveniente das folhas de guapuruvu ( $S$. parahyba), fedegoso ( $S$. macranthera), capixingui (C.floribundus) e cutieira (J. princeps) depositadas no solo ao longo de um ano (agosto 2005 - julho 2006).

Figure 7 - Magnesium input from leaves of guapuruvu ( $S$. parahyba), fedegoso (S. macranthera), capixingui (C. floribundus) and cutieira (J. princeps) deposited on soil over one year (August 2005-July 2006).

\section{CONCLUSÕES}

As árvores de cutieira e capixingui apresentaram alta capacidade de aporte nutrientes através da serapilheira, durante a época de alta demanda de nutrientes dos cafeeiros. Devem ser estudadas outras características das folhas como o teor de lignina e polifenóis e a taxa de decomposição e liberação dos nutrientes, para avaliar a verdadeira capacidade de disponibilização de nutrientes para a cultura.

O aporte de massa de folhas e de nutrientes do guapuruvu foi pouco importante dentro do SAF. Estudos sobre a capacidade da serapilheira proveniente desta espécie de conservar a umidade do solo durante o inverno podem ser desenvolvidos com mais detalhe.

Apesar do fedegoso apresentar baixo aporte de nutrientes quando comparado com as outras espécies do SAF, a grande deposição de massa na época de alta demanda de nutrientes dos cafeeiros fazem dele uma espécie potencial para ser usada nos SAF com café da região.

R. Árvore, Viçosa-MG, v.32, n.5, p.869-877, 2008 


\section{AGRADECIMENTOS}

Agradecemos ao agricultor Ilson Jose de Medeiros Lopes por sua participação, ao CNPq pelo suporte financeiro e à Universidade Federal de Viçosa pelo suporte físico e técnico.

\section{REFERÊNCIAS}

AIDAR, M. P. M.; JOLY, C. A. Dinâmica da produção e decomposição de serapilheira do araribá (Centrolobium tomentosum Guill. ex Benth.- Fabaceae) em uma mata ciliar, Rio JacaréPepira, São Paulo. Revista Brasileira de Botânica, v.26, n.2, p.193-202, 2003.

AUGUSTO, D. C. C. et al. Utilização de esgotos domésticos tratados através de um sistema biológico na produção de mudas de Croton floribundus Spreng. (Capixingui) e Copaifera langsdorffii Desf. (Copaíba). Revista Árvore, v.27, n.3, p.335-342, 2003.

ASSOCIATION OF OFFICIAL ANALYTICAL CHEMISTIC - AOAC. Official methods of analysis of the association of official analytical chemists. 12.ed. Washington: 1975. 1094p.

ARATO, H. D.; MARTINS, S. V.; FERRARI, S. H. S. Produção e decomposição de serapilheira em um sistema agroflorestal implantado para recuperação de área degradada em Viçosa- MG. Revista Árvore, v.27, n.5, p.715-721, 2003.

BACON, C. Confronting the coffee crisis: can fair trade, organic, and specialty coffees reduce small-scale farmer vulnerability in northern Nicaragua? World Development, v.33, n.3, p.497-511, 2005.

BRAGA, J. M.; DEFELIPO, B. V. Determinação espectofotométrica de $\mathrm{P}$ em extratos de solo e material vegetal. Revista Ceres, v.21, n.113, p.73-85, 1974.

BYARD, R.; LEWIS, K. C.; MONTAGNINI, F. Leaf litter decomposition and mulch performance from mixed and monospecific plantations of native tree species in Costa Rica. Agriculture,

Ecosystems and Environment, v.58, n.2/3, p.145-155, 1996.

R. Árvore, Viçosa-MG, v.32, n.5, p.869-877, 2008
CAMPANHA, M. M. et al. Growth and yield of coffee plants in agroforestry and monoculture systems in Minas Gerais, Brazil. Agroforestry Systems, v.63, n.1, p.75-82, 2004.

FLORES-AYLAS, W. W. et al. Efeito do Glomus etunicatum em fósforo no crescimento inicial de espécies arbóreas em semeadura direta.

Pesquisa Agropecuária Brasileira, v.38, n.2, p.257-266, 2003.

FRANCO, F. S. et al. Quantificação de erosão em sistemas agroflorestais e convencionais na Zona da Mata de Minas Gerais. Revista Árvore, v.26, n.6, p.751-760, 2002.

GIRALDO, J.; JARAMILLO, R. Ciclo hodrilógico y transporte de nutrimentos en cafetales bajo diferentes densidades de sombrio de guamo. Cenicafé, v.55, n.1, p.52-68, 2004.

GINDABA, J.; ROZANOV, A.; NEGASH, L. Trees on farms and their contribution to soil fertility parameters in Badessa, eastern Ethiopia.

Biology and Fertility of Soils, v.42, n.1, p.66-71, 2005.

GONÇALVES, J. L. Produção de mudas de espécies nativas: substrato, nutrição, sombreamento e fertilização. In: GONZALVES, J. L. M.; BENENDETTI, V. (Eds.) Nutrição e fertilização flrestal. Piracicaba: IPEF, 2000. p.179-195.

HAIRIAH, K. et al. Litter layer residence time in forest and coffee agroforestry systems in Sumberjaya, West Lampung. Forest Ecology and Management, v.224, n.1, p.45-57, 2006.

JOHNSON, C. M.; ULRICH, A. Analytical methods for use in plant analysis. Los Angeles: University of California, 1959. 247p.

KINAMA, J. M. et al. Evaporation from soils below sparse crops in contour hedgerow agroforestry in semi-arid Kenya. Agricultural and Forest Meteorology, v.130, n.3/4, p.149-162, 2005.

LEBLANC, H.A.; McGRAW, R.L.; NYGREN, P.; Le ROUX, C. Neotropical legume tree Inga edulis forms $\mathrm{N}_{2}$-fixing symbiosis with fast-growing Bradyrhizobium strains. Plant and Soil, v.275, p.123-133, 2005. 
LIVESLEY, S. J.; GREGORY, P. J.; BURESH, R. J. Competition in tree row agroforestry systems. 1 . Distribution and dynamics of fine root length and biomass. Plant and Soil, v.227, n.1/2, p.149-161, 2000.

LODHIYAL, N.; LODHIYAL, L. S.; PANGTEY, Y. P. S. Structure and function of Shisham forest in central Himalaya, India: nutrient dynamics.

Annals of Botany, v.89, n.1, p.55-65, 2002.

LODHIYAL, N.; LODHIYAL, L. Nutrient cycling and nutrient use efficiency in short rotation, high density Central Himalayan Tarai Poplar plantations. Annals of Botany, v.79, n.5, p.517-527, 1997.

MARTINS, F. R. Estrutura de uma Floresta Mesófila. Campinas: UNICAMP, 1991. 246p.

MARTINS, S. V.; RODRIGUES, R. R. Produção de serapilheira em clareiras de uma floresta estacional semidecidual no município de Campinas, SP. Revista Brasileira de Botânica, v.22, n.3, p.405-412, 1999.

MENDONÇA, E. S.; STOTT, D. E.

Characteristics and decomposition rates of pruning residues from a shaded coffee system in Southeastern Brazil. Agroforestry Systems, v.57, n.2, p.117-125, 2003.

MUSVOTO, C.; CAMPBELL, B. M.;

KIRCHMANN, H. Decomposition and nutrient release from mango and miombo woodland litter in Zimbabwe. Soil Biology \& Biochemistry, v.32, n.8/9, p.1111-1119, 2000.

NEVES, Y. P. Evolução da fertilidade do solo, retenção de umidade, crescimento vegetativo, produção e teores foliares de nutrientes em cafeeiros cultivados a pleno sol e consorciados. 2001. 65f. Dissertação (Mestrado emFitotecnia) - Universidade Federal de Viçosa, Viçosa, MG, 2001.

NYGREN, P.; RAMÍREZ, C. Production and turnover of $\mathrm{N}_{2}$ fixing nodules in relation to foliage development in periodically pruned Erythrina poeppigiana (Leguminosae) trees. Forest Ecology and Management, v.73, n.1/3, p.59-73, 1995.
PARROTTA, J. A. Productivity, nutrient cycling, and succession in single- and mixes-species plantations of Casuarina equisetifolia, Eucalyptus robusta, and Leucaena leucocephala in Puerto Rico. Forest Ecology and Management, v.124, n.1, p.45-77, 1999.

PAULA, S. A.; LEMOS FILHO, J. P. Dinâmica do dossel em mata semidecidua no perímetro urbano de Belo Horizonte, MG. Revista Brasileira de Botânica, v.24, n.4, p.545-551, 2001.

PERFECTO, I. et al. Biodiversity, yield and shade coffee certification. Ecological Economics, v.54, n.4, p.435-446, 2005.

SCHROTH, G. et al. Plant-soil interactions in multistrata agroforestry in the humid tropics. Agroforestry Systems, v.53, n.1, p.85-102, 2001.

SCHUMACHER, M. V. et al. Produção de serapilheira em uma floresta de Araucária angustifolia (Bertol) Kuntze no município de Pinhal Grande - RS. Revista Árvore, v.28, n.1, p.29-37, 2004.

SCHWENDENER, C. M. et al. Nitrogen transfer between high- and low-quality leaves on a nutrient-poor Oxisol determined by ${ }^{15} \mathrm{~N}$ enrichment. Soil Biology \& Biochemistry, v.37, n.4, p.787-794, 2005.

SENWAL, R. L. et al. Leaf litter decomposition and nutrient release patterns of six multipropose tree species of central Himalaya, India. Biomass and Bioenergy, v.24, n.1, p.3-11, 2003.

SILVA, E. A. et al. Seasonal changes in vegetative growth and photosynthesis of Arabica coffee trees. Field Crops Research, v.89, n.2/3, p.349-357, 2004.

SOUZA, H. N. et al. Sistemas agroflorestais e a sustentabilidade da agricultura familiar na Zona da Mata de Minas Gerais. Boletim Centro de Tecnonogias Alternativas da Zona da Mata - CTA, n.2, p.11, 2006.

VILELA, D. M. et al. Effect of selective logging on forest structure and nutrient cycling in a seasonally dry Brazilian Atlantic forest. Journal of Biogeography, v.33, n.3, p.506-516, 2006.

R. Árvore, Viçosa-MG, v.32, n.5, p.869-877, 2008 